Service social

\title{
La qualité. Sa définition et sa mesure
}

\section{Jacques Plante et Chantal Bouchard}

Volume 47, numéro 1-2, 1998

Évaluation - Colloque 1999

URI : https://id.erudit.org/iderudit/706780ar

DOI : https://doi.org/10.7202/706780ar

Aller au sommaire du numéro

Éditeur(s)

École de service social de l'Université Laval

ISSN

1708-1734 (numérique)

Découvrir la revue

Citer cet article

Plante, J. \& Bouchard, C. (1998). La qualité. Sa définition et sa mesure. Service social, 47(1-2), 27-61. https://doi.org/10.7202/706780ar
Résumé de l'article

La qualité est présentée ici comme un idéal vers lequel nous tendons sans pouvoir l'atteindre. La principale difficulté qu'éprouvent les personnes soucieuses de la mesurer tient au fait qu'elles se réfèrent le plus souvent à une qualité universelle et globale diffuse et mal définie. Ce problème s'explique également par la difficulté d'en déterminer des indicateurs qui peuvent validement témoigner de la qualité. Après avoir jeté un regard rapide sur l'histoire qui a mené à l'engouement actuel pour la qualité, les auteurs présentent ici un modèle holistique à l'intérieur duquel la qualité globale est éclatée en neuf qualités spécifiques. Il est présumé dans ce modèle que ces neuf qualités spécifiques conviennent à l'ensemble des organisations, des programmes, des services et des interventions. Ces qualités sont définies de manière opérationnelle et mutuellement exclusive afin de se prêter plus facilement à la mesure. Le but premier de ce modèle est d'abord de servir de guide lorsqu'il s'agit de déterminer des indicateurs de la qualité, mais aussi de réfèrent à la mesure de la qualité et à son évaluation.
Ce document est protégé par la loi sur le droit d'auteur. L'utilisation des services d’Érudit (y compris la reproduction) est assujettie à sa politique d'utilisation que vous pouvez consulter en ligne.

https://apropos.erudit.org/fr/usagers/politique-dutilisation/ 


\section{La qualité \\ Sa définition et sa mesure}

Jacques PLANTE
Chantal BOUCHARD

La qualité est présentée ici comme un idéal vers lequel nous tendons sans pouvoir l'atteindre. La principale difficulté qu'éprouvent les personnes soucieuses de la mesurer tient au fait qu'elles se réfèrent le plus souvent à une qualité universelle et globale diffuse et mal définie. Ce problème s'explique également par la difficulté d'en déterminer des indicateurs qui peuvent validement témoigner de la qualité. Après avoir jeté un regard rapide sur l'histoire qui a mené à l'engouement actuel pour la qualité, les auteurs présentent ici un modèle holistique à l'intérieur duquel la qualité globale est éclatée en neuf qualités spécifiques. Il est présumé dans ce modèle que ces neuf qualités spécifiques conviennent à l'ensemble des organisations, des programmes, des services et des interventions. Ces qualités sont définies de manière opérationnelle et mutuellement exclusive afin de se prêter plus facilement à la mesure. Le but premier de ce modèle est d'abord de servir de guide lorsqu'il s'agit de déterminer des indicateurs de la qualité, mais aussi de référent à la mesure de la qualité et à son évaluation.

Quality is presented here as an ideal we strive to attain, without being able to do so. The major problem encountered by those who wish to measure it lies in the fact that, most often, their reference is a universal, overall, vague and ill-defined quality. The problem can also be explained by the difficulty in determining valid criteria that can be used to evaluate quality. The authors present here a holistic model within which global quality is broken down into nine specific components. In this model, it is assumed that these nine qualities apply to all organizations, programs, services and actions. They are defined in an operational and mutually exclusive manner in order to be more easily measurable. The prime objective of this model is to serve as a guide in determining the criteria for quality, but also, as a point of reference in measuring quality and evaluating it. 
Aujourd'hui, s'il est un sujet qui suscite de vives discussions dans les établissements qui offrent des programmes ou des services, c'est bien celui de la qualité. Plus précisément, c'est celui de la mesure de la qualité et de son évaluation qui retient le plus l'attention.

La qualité est sans conteste le concept le plus utilisé comme objet de promotion de services et de programmes. II suffit, pour nous en convaincre, de penser à la multiplicité des services à la clientèle et aux divers sondages auprès de la population pour recueillir son opinion. Sans tenir compte du rapprochement parfois insidieux entre la " satisfaction de la clientèle " et la " qualité ", il demeure selon Reeves et Bednar (1994) que la qualité est sans nul doute le mantra le plus souvent répété parmi les gestionnaires et les chefs d'entreprise.

Les raisons de cette quête de la qualité sont diverses. Tantôt elle est imposée par les exigences de la clientèle. Tantôt elle s'explique par les lois de la concurrence qui font que les consommateurs s'orientent vers les lieux où les biens et les services sont les meilleurs. Souvent aussi, le souci de la qualité peut être inhérent au mandat confié aux établissements ou encore prendre son origine dans une volonté organisationnelle d'œuvrer dans des conditions idéales pour offrir aux consommateurs ou aux usagers les meilleurs services ou programmes possible.

L'engouement pour la qualité s'explique également par d'autres causes. Parmi celles-là, mentionnons le changement de la nature du travail, les initiatives particulières pour améliorer les façons de faire, les reconnaissances nationales et internationales, les changements dans les rôles qu'assument les organismes dans leur milieu, les modifications dans la demande ainsi que le pouvoir des technologies de l'information (Neely, 1999). Nous pouvons ajouter à cette énumération la prise de conscience des clients à l'égard du droit d'exiger le meilleur ou, à tout le moins, ce qui est conforme à leurs attentes.

\section{LA QUALITÉ : TROIS DÉFIS À RELEVER}

Malgré le fait que la qualité semble une réalité difficile à matérialiser, il n'en demeure pas moins que plusieurs désirent la gérer pour mieux l'atteindre. Des auteurs tels Deming (1986), Oakland (1993) et Morrison (1994) affirment à ce propos qu'il est impossible de gérer ce qui ne peut être mesuré. À cela nous pourrions ajouter qu'il est tout autant impossible de mesurer ce qui n'est pas clairement défini. II est difficile 
de croire en effet que l'on peut faire l'économie de la définition de la qualité et prétendre vouloir mesurer celle-ci pour mieux l'atteindre. La tâche ne semble pas facile à première vue, puisque les nombreuses définitions qu'on en donne laissent croire qu'il s'agit là d'un terme polysémique, voire ambigu.

Trois défis se présentent donc aux décideurs et aux gens de terrain qui se préoccupent de la qualité : sa définition et sa mesure, la détermination de ses indicateurs, son évaluation.

Dans le présent article nous nous intéressons au premier de ces trois défis. Nous clarifierons d'abord les divers concepts utiles à la mesure de la qualité. Nous proposerons ensuite un modèle holistique décrivant les composantes spécifiques de la qualité. Le but de ce modèle est de servir de guide à la production des indicateurs utilisés dans la mesure de la qualité. II va de pair avec la question de la vérification continue de la qualité, partout où elle se pose. Étant donné la nature particulière de ce premier défi, il ne faudra pas s'étonner du contenu quelque peu théorique de cet article'.

\section{VERS UN MODÈLE SCHÉMATIQUE DE RÉFÉRENCE DE LA MESURE DE LA QUALITÉ}

\section{Les termes à clarifier}

\section{La mesure}

ou la quantification de l'objet ou du phénomène observé

C'est faire preuve de truisme que d'affirmer que nous ne pouvons mesurer que ce qui a été préalablement défini. La mesure, en effet, s'oppose au flou et sa qualité tient à la clarté de la définition de l'objet ou du phénomène à observer. Dans le domaine de la mesure de la qualité, des termes prêtent à confusion à un point tel qu'en certaines circonstances nous revenons au moment de la construction de la tour

1. Dans un deuxième article, plus pragmatique celui-là, à paraître par ailleurs ultérieurement, nous tenterons d'aider les personnes désireuses de relever le défi de la production des indicateurs de la qualité. Dans le troisième et dernier article de cette série, nous examinerons deux questions particulières. Celle d'abord de la robustesse des indicateurs de la qualité, puis celle de l'évaluation même de la qualité. 
de Babel. Qu'est-ce que la mesure? Peut-on la distinguer de l'évaluation? Qu'est-ce que la qualité? À l'image du précepte de Descartes, peut-on la décomposer en ses composantes principales les plus simples de façon à rendre la mesure plus facile? Qu'est-ce qu'un indicateur de qualité et un indicateur de la qualité? Voilà quelques-unes des questions incontournables auxquelles nous tenterons de répondre dans le présent article.

En ce qui concerne la mesure de la qualité, le premier terme à clarifier est celui de la mesure elle-même. Mesurer, nous rappelle Plante, « c'est attribuer une quantité quelconque à un phénomène ou à un objet, à partir d'une règle d'attribution déterminée a priori » (1994, p. 18). La mesure se distingue en cela de l'évaluation qui, elle, consiste à porter un jugement de valeur en comparant les données obtenues à la suite de la mesure avec des critères, des normes ou des standards.

La qualité d'une mesure exige d'abord une définition claire et précise de l'objet visé. Cette définition, pour répondre aux conditions de la clarté, doit permettre de distinguer cet objet de tout autre objet qui peut lui ressembler ou avec lequel il peut être confondu. C'est ainsi qu'on utilise une échelle à intervalles sur un thermomètre pour quantifier la température et en distinguer les degrés, le gramme pour exprimer la valeur des différents poids et le centimètre pour préciser différentes longueurs. Le choix de l'outil de mesure est déterminé à la fois par la nature même de l'objet visé ou du phénomène observé et par la ou les propriétés propres à cet objet ou à ce phénomène. Ainsi, on n'utilisera pas un thermomètre pour déterminer la longueur d'une table, pas plus que l'on ne fera appel à une balance pour exprimer sa largeur.

La clarté de la définition de l'objet visé ne suffit pas pour assurer la qualité de la mesure. Pour rendre compte de la réalité observée, celleci doit posséder au moins deux caractéristiques (Plante, 1994). Elle doit d'abord donner le même résultat lorsqu'elle est répétée dans les mêmes conditions, avec l'aide du même instrument. II s'agit ici d'une caractéristique de la mesure appelée la fidélité. Cette dernière caractéristique, tout en étant essentielle, n'est pas à elle seule satisfaisante. Une mesure en effet peut être fidèle sans que les résultats qu'elle fournit correspondent pour autant à la réalité. Ce pourrait être le cas par exemple d'un indicateur de vitesse dont l'échelle de graduation est faussée. Les informations qu'un tel indicateur présente à un observateur sont fidèles, mais elles ne sont pas valides. La validité est 
une autre caractéristique que doit impérativement posséder un instrument de mesure et qui correspond à la capacité que possède un instrument de mesurer ce qu'il prétend mesurer.

\section{La qualité}

ou la conformité avec ce qui est désiré à un moment donné

Qu'est-ce donc en effet que la qualité? Après avoir répertorié un grand nombre de publications sur le sujet, Neergaard (1999) en arrive à la conclusion que le concept de qualité demeure vague, ce qui en rend la mesure et l'évaluation fort problématiques. Comme le note avec humour Peter Cusins (1994), "j'ignore comment définir la qualité, mais je le sais lorsqu'elle fait défaut ».

Lorsqu'il s'agit de définir la qualité, le terme qui semble le mieux convenir pour en révéler la nature intrinsèque est celui de « conformité ". Ce terme est intéressant dès lors qu'il renvoie à l'existence d'au moins deux éléments. Un premier sert de modèle de référence ou de standard, un second lui est comparé afin d'en mesurer le degré de correspondance ou de ressemblance et de juger de sa valeur. Nous retrouvons ici les opérations premières de la mesure et de l'évaluation de la qualité qui consistent à quantifier d'abord, à comparer ensuite. Nous rejoignons aussi par là la position traditionnelle signalée par Neely et collab. (1995) lorsqu'il s'agit de définir la qualité; celle-ci étant considérée comme la conformité avec des spécifications préalablement définies par les demandeurs ou les usagers.

La difficulté dont nous font part les auteurs lorsqu'il s'agit de circonscrire le concept de qualité tient au fait que nous nous référons le plus souvent à une qualité universelle et globale convenant à l'ensemble des établissements, comme à l'ensemble des programmes et services. Il apparaît que nous devrions plutôt parler de qualités circonstanciées ou spécifiques, signifiant ainsi qu'il s'agit pour un établissement de rechercher non pas " la » qualité, mais des qualités attendues.

Selon Plante, la qualité doit se définir comme un idéal vers lequel doit tendre un établissement, considéré dans sa totalité ou à travers l'une ou l'autre de ses parties. La qualité, dit-il, demeure une fin que nous ne connaissons pas, mais vers laquelle nous tendons par approximations successives, au fur et à mesure que des informations nouvelles sont portées à notre connaissance. Chaque approximation constitue 
une hypothèse de la qualité à un moment donné, pour autant qu'elle soit conforme aux informations dont on dispose à ce moment (1994, p. 29). Vue sous cet angle, ajoute-t-il, un établissement amélioré ne demeure qu'une approximation plus fine de la qualité idéale vers laquelle elle doit tendre.

Selon toute apparence, la qualité vue dans sa globalité est un concept pluriel pour lequel nous trouvons une résonance particulière chez chacun de ses utilisateurs. Les expressions utilisées pour la décrire rendent bien compte de ces résonances. Peters et Waterman (1982) définissent la qualité comme étant l'excellence, d'autres comme une valeur (Feigenbaum, 1961), conforme à l'utilisation voulue (Juran et Gryna, 1988), conforme aux spécifications (Gilmore, 1974) ou à ce qui est requis (Crosby, 1979), comportant zéro défaut (Crosby, 1979) qui satisfait ou dépasse les exigences des consommateurs (Parasuraman et collab., 1985) et autres. II se dégage de l'ensemble de ces expressions que les trois points de vue les plus souvent adoptés par ces auteurs, lorsqu'ils font référence à la qualité, c'est celui de conformité avec ce qui est voulu ou désiré. (Crosby, 1979; Gilmore, 1974), celui de conformité avec ce qui est perçu (Parasuraman et collab., 1985) et, enfin, celui de conformité avec ce qui est effectivement produit (Feigenbaum, 1961; Crosby, 1979).

\section{Les trois points de vue à adopter pour la mesure de la qualité}

II semble, affirme Cusins (1994), qu'il existe au moins deux types de qualités : un premier type que l'on pourrait qualifier de statique et dont la présence ou l'absence est facile à constater; et un deuxième que l'on pourrait désigner comme dynamique et qui crée une impression de haute qualité.

Neergaard (1999) fait également allusion à cette impression ou, pourrait-on dire, à cette perception de la qualité par opposition à la description de la qualité elle-même, cette dernière renvoyant plutôt à ce qui est désiré comme qualité et à ce qui est effectivement produit. II mentionne que les trois types de description de la qualité les plus signalés dans les 270 études consultées sont la qualité désirée, la conscience de la qualité et la conformité au standard de la qualité. 
Le Mouvement français pour la qualité (1994) stipule dans son ouvrage Document synthèse du groupe de travail - indicateur qualité et tableau de bord qu'un établissement, un programme, un service possède une qualité interne, mais également une qualité qui relève du perçu.

Plante (1994), enfin, au terme d'une revue de nombreux ouvrages spécialisés dans le domaine de la mesure de la qualité, en arrive à la conclusion que la qualité doit être mesurée en adoptant trois points de vue interreliés :

- celui de la qualité voulue, qui est révélée ou annoncée dans les objectifs visés;

- celui de la qualité rendue qui se matérialise pendant son déroulement, à travers les ressources, les acteurs, les résultats et les effets;

- et celui de la qualité perçue, c'est-à-dire ce que ressentent les consommateurs, les usagers, les pourvoyeurs et les autres personnes intéressées.

Tous ces points de vue se révèlent d'égale importance. Ils se conjuguent et s'harmonisent au moment où un service est destiné à une clientèle. Alors que la qualité voulue et la qualité rendue font depuis longtemps partie de l'attention des gestionnaires, la préoccupation plus tardive de ceux-ci à l'égard de la satisfaction de la clientèle relève de la prise de conscience de l'importance de la perception des consommateurs de services. Plante (1994) insiste sur l'interdépendance du voulu, du rendu et du perçu. Ainsi, la qualité rendue doit à la fois correspondre à la qualité voulue et être bien perçue.

\section{La confusion autour des termes satisfaction et contentement}

Au début de cet article, nous avons signalé que le management de la qualité prenait comme base les besoins des usagers et la satisfaction de ces besoins. Lorsqu'il s'agit de mesurer cette satisfaction, il faut veiller à ce que l'objet ou les objets à l'égard desquels on désire connaître le degré de satisfaction correspondent à ce qui est voulu et à ce qui est rendu.

Harte et Dale (1995) distinguent le concept de satisfaction de la clientèle selon qu'il s'applique à des entreprises de production et de fabrication de produits ou de services tangibles ou qu'il s'applique à des 
organismes de services professionnels. Dans le premier cas, la satisfaction de la clientèle constitue le principal, sinon le plus important des objectifs visés. Dans le second cas, les objectifs des services effectivement donnés visent le plus souvent la satisfaction des besoins de la personne ou des besoins de la société, du milieu ou de la collectivité.

Le concept de besoin se définit ici en référence à un manque chez la personne. II peut correspondre à un désir et il se matérialise souvent sous forme de demande. Cependant, une demande ou un désir peut ne pas correspondre à un besoin, pouvant même être incompatible avec un besoin. Par exemple, une personne dont la santé est déficiente peut formuler des demandes ou manifester des désirs à l'encontre de son besoin de santé.

Le fait de se préoccuper en priorité de la satisfaction de la clientèle risque dans ces circonstances de donner lieu à une confusion entre le concept de "satisfaction " des besoins de la clientèle et celui de « contentement » de la clientèle.

Pour illustrer ce propos, imaginons un établissement dont la raison d'être est de répondre aux besoins de santé des usagers qui font appel à ses services. Une étude de « satisfaction » menée auprès de personnes obèses souffrant de diabète nous révèle que ces personnes expriment un haut degré d'insatisfaction à l'égard de la qualité de nourriture que leur offre l'établissement qu'elles fréquentent. Ces personnes sont d'avis que la nourriture est fade et, par conséquent, elles souhaitent que l'établissement en améliore la qualité en y ajoutant des condiments.

Le perçu, avons-nous dit plus haut, doit s'harmoniser avec la qualité voulue et la qualité rendue. Manifestement, dans cet exemple, le voulu a trait aux besoins de santé des personnes. Le rendu quant à lui se matérialise sous la forme du service d'alimentation qui prépare le menu des personnes concernées. Le perçu, pour être en harmonie avec le voulu et le rendu, doit donc impliquer un jugement sur la satisfaction des besoins des personnes qui reçoivent le service.

Dans l'exemple, le jugement des personnes consultées n'est pas lié aux besoins de santé. II s'agit plutôt ici de l'expression d'un degré de contentement des personnes à l'égard de la nourriture. C'est là une donnée certes importante pour l'établissement concerné, mais le mécontentement manifesté ici ne doit pas être interprété comme une 
insatisfaction des besoins en cause. II y a lieu de croire qu'il ne s'agit pas d'une qualité perçue de l'établissement, celle-ci devant se juger au regard de la raison d'être de l'établissement, mais plutôt d'une mesure du contentement de la clientèle qui probablement relève d'une autre préoccupation que celle de la qualité perçue de l'établissement.

Lorsqu'il s'agit de mesurer la qualité perçue à partir d'une étude de la satisfaction de la clientèle à l'égard d'un service professionnel, il est important de veiller à ce que les divers éléments soumis au jugement des personnes soient en lien direct avec ce qui est voulu et rendu.

Dans le cas d'un service d'accueil dans un établissement, il peut être justifié de mener une étude de la satisfaction - entendons ici le contentement -, au regard d'éléments tels le confort des sièges, la couleur des murs ou la décoration, parce que ces éléments ont un lien de cohérence avec ce qui est voulu et rendu dans ce service d'accueil.

Soumettre de tels éléments au jugement des usagers, lorsqu'il s'agit de mesurer la qualité d'un service professionnel en éducation, en santé ou dans un autre domaine, est plus difficile à justifier parce que cela n'a pas de lien de cohérence avec ce qui est voulu et rendu. Dans ce dernier cas, il s'agit davantage d'une intention de connaître une forme particulière de satisfaction dont le nom le plus exact devrait être le « contentement ».

\section{Les éléments constituants de la qualité globale}

La qualité, avons-nous dit, peut être considérée comme la conformité d'un objet ou d'un phénomène à des spécifications préalablement définies par des demandeurs (société, milieu, collectivité et individus), des concepteurs ou des spécialistes.

Dans cette perspective, le problème principal reste de déterminer les éléments au regard desquels le degré de conformité, c'est-à-dire la qualité, doit être mesuré et de représenter ces éléments les uns par rapport aux autres en un modèle holistique qui rend compte de la qualité globale à un moment donné.

Divers éléments sont proposés à titre de références pour mesurer la qualité globale. Brunelle (1993) est d'avis que la qualité s'établit en fonction des résultats obtenus, en tenant compte des ressources investies et de ce qu'il est possible de réaliser dans un contexte donné. 
II insiste sur le fait que la qualité ne doit pas se confondre avec la structure d'une organisation, ses façons de faire, c'est-à-dire ses processus, et ses résultats. Elle se situe uniquement dans les liens entre ces éléments. À l'instar de plusieurs auteurs, Saucier et Brunelle (1995) identifient plusieurs éléments pouvant servir de base pour appréhender la qualité. Ils signalent notamment l'efficacité, l'efficience, la productivité et la pertinence. De leur côté, Harte et Dale (1995) font référence à une organisation empathique, c'est-à-dire ajustée aux clients. Brunelle (1993), quant à lui, juge de la qualité d'une organisation en termes de liens de conformité avec une clientèle cible, relativement à des effets directs (prévus) et indirects (non prévus) dans un environnement physique et social.

En tenant compte de l'ensemble des éléments qui peuvent être considérés au moment de procéder à la mesure de la qualité d'un établissement, Plante (1994) en arrive de son côté à la conclusion qu'il serait plus simple de considérer qu'un établissement, un programme ou un service possède une qualité globale, qu'il définit comme un idéal à atteindre et qui peut être éclatée sous la forme de plusieurs qualités spécifiques se prêtant davantage à la mesure.

En s'appuyant sur divers travaux consultés dans le domaine, Plante (1994) a identifié, en 1994, huit éléments spécifiques associés chacun à la qualité globale. C'est au regard de ces éléments que le degré de conformité doit être mesuré afin que l'objet examiné (établissement, programme, service, intervention, etc.) puisse se prêter à l'évaluation de la qualité. Plante a par la suite représenté sous la forme d'un modèle les liens de conformité devant exister entre ces éléments.

Dans ce modèle, chaque lien de conformité porte un nom particulier. Ces noms désignent autant de qualités spécifiques qui peuvent être associées à la qualité globale de l'objet examiné. Ils conviennent également autant au point de vue macroscopique qu'au point de vue microscopique qu'une personne peut adopter face à cet objet. Chacun des liens ainsi nommés peut être défini de manière mutuellement exclusive à tout autre lien figurant dans le modèle, ce qui pour Plante constitue une condition nécessaire à la mesure.

Les premières ébauches de ce modèle schématique de la qualité globale ont été possibles grâce à la collaboration de certains praticiens de l'évaluation de programmes. Des versions initiales successives de 
ce modèle ont été publiées dans plusieurs documents (Commissariat général du plan et École nationale d'administration [ENA] à Paris, 1991; Dictionnaire actuel de l'éducation, 1993; Plante, 1994; Unesco, 1995, etc). Depuis, des travaux réalisés en collaboration avec $\mathrm{M}^{\mathrm{me}}$ Chantal Bouchard, responsable du développement au Conseil québécois d'agrément d'établissements de santé et de services sociaux, ont permis d'améliorer significativement ce schéma.

Dans sa version actuelle, Plante et Bouchard proposent donc un modèle où la qualité globale est éclatée sous la forme de neuf éléments. Ces éléments de référence sont incontournables, peu importe le type d'établissement ou d'entreprise et peu importe le type d'intervention, son ampleur ou sa nature.

Le but premier de ce modèle schématique est de servir de référentiel à la production d'indicateurs de la qualité d'un établissement. II ne faudra donc pas s'étonner de constater que tous les liens indiqués dans le modèle sont focalisés sur l'établissement qui est ici considéré comme le « producteur " principal de la qualité.

En reprenant l'argument déjà évoqué qu'il est impossible de mesurer un objet qui n'a pas été préalablement défini, nous devons, avant même de présenter le modèle, définir l'établissement qui en constitue le cœur. C'est par le truchement de cette définition que nous pourrons le mieux faire le pont entre les considérations théoriques propres à la qualité et la réalité du terrain où se situe cet établissement.

\section{La définition d'un établissement}

La définition d'un d'établissement est sensiblement la même que celle que l'on pourrait donner à un programme, un service, une intervention et un projet. Les mêmes éléments caractéristiques s'y retrouvent. II suffit de substituer au terme établissement utilisé dans la définition qui suit le terme qui convient le mieux à la situation.

Un établissement peut se définir comme un ensemble probabiliste qu'il faut sans cesse remettre en question afin d'améliorer son parcours. Cela se traduit par un examen attentif de son fonctionnement général et de chacune de ses parties significatives. Vu sous un autre angle, un établissement doit également être considéré comme un outil de changement. La définition d'un établissement doit donc inclure non seulement les éléments qui le composent, mais aussi sa raison d'être 
et les personnes responsables d'assurer la qualité (administrateurs, cadres, employés, intervenants etc.)

L'établissement peut se définir comme un ensemble cohérent, organisé et structuré d'objectifs, de moyens et de personnes qui l'animent. II a pour raison d'être des besoins manifestés ou anticipés dans la société, le milieu ou la collectivité. Il peut être modifié par des personnes responsables qui possèdent une marge de manœuvre suffisante pour assurer la qualité de son fonctionnement.

L'ensemble cohérent, organisé et structuré dont il est question ici s'oppose à l'existence d'un rassemblement d'agrégats divers. Par cohérent, organisé et structuré, il faut entendre la possibilité d'expliquer la présence d'un élément au sein de l'établissement par la présence d'un autre élément qui le précède. Ainsi, les objectifs se justifient sur la base des besoins qui les précèdent, les moyens, sur la base des objectifs et les personnes qui y travaillent sur la base individuelle ou combinée des moyens et des objectifs

Les objectifs, pour leur part, doivent être considérés comme des résultats à atteindre. Ils peuvent être généraux ou particuliers, mais c'est sous cette dernière forme, en raison de leur caractère davantage opérationnel, que l'on peut le mieux justifier l'utilisation des moyens déterminés pour les atteindre.

Le terme moyen, quant à lui, fait référence à tout ce à quoi on fait appel pour atteindre les objectifs visés. En font partie les stratégies, les activités, les méthodes, les locaux, les outils, les budgets consentis, etc. Tout comme il est logique de croire que le mot chien n'a jamais mordu personne, il est tout aussi logique de penser qu'un établissement n'existe pas que par son descriptif sur papier. C'est une entité vivante et, à ce titre, ce sont les personnes qui y travaillent qui lui fournissent les caractéristiques fonctionnelles et qui en font un outil de changement et de qualité.

Enfin, condition incontournable d'amélioration, l'établissement doit posséder en son sein des personnes dont la marge d'action est suffisante pour prendre les décisions qui s'imposent et apporter les changements qui se révéleraient nécessaires à un meilleur fonctionnement. 
Mesure de la qualité

Schéma des neuf composantes

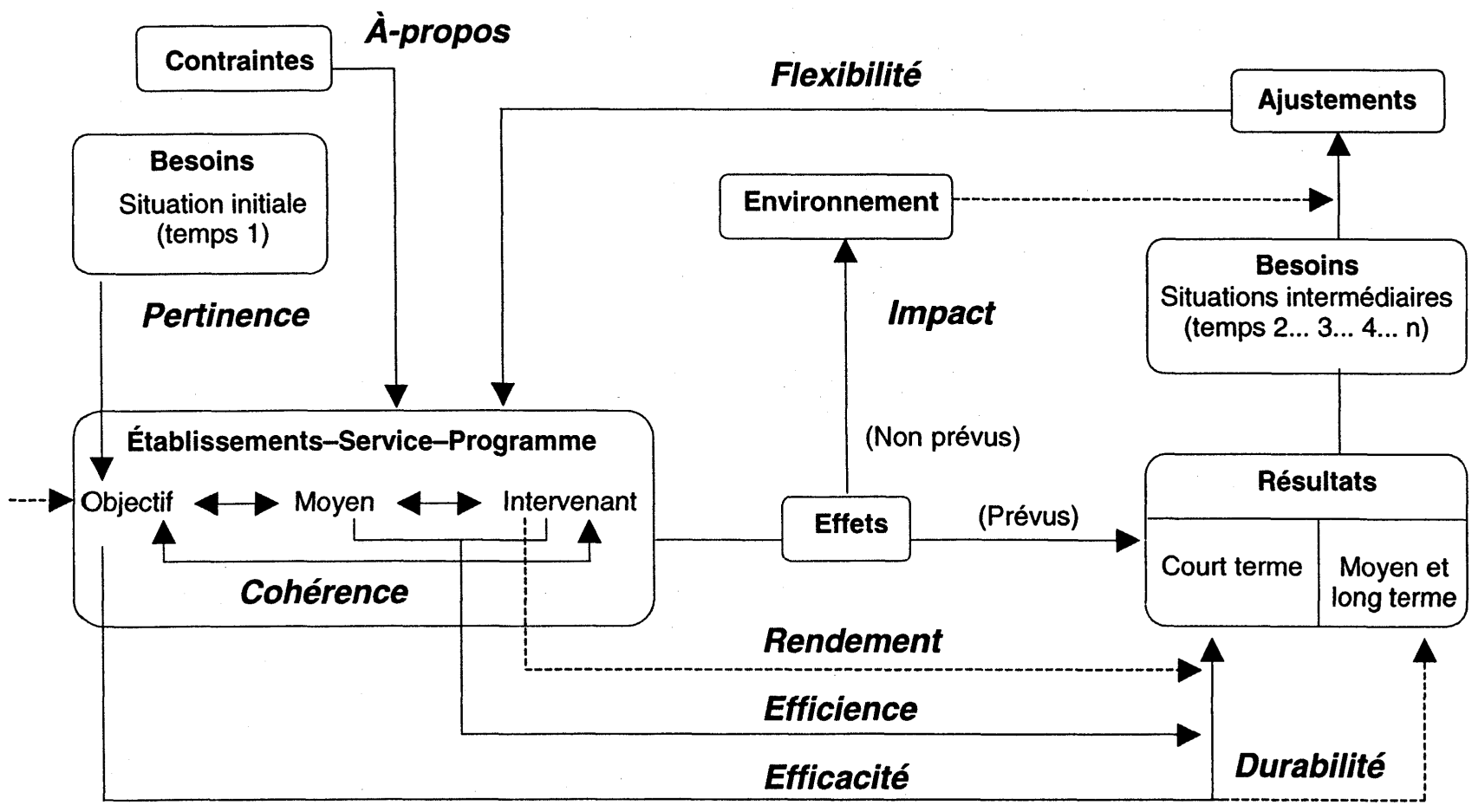




\section{La définition des neuf qualités spécifiques comprises dans le modèle schématique de référence de la qualité globale}

À la base, nous considérons dans ce modèle schématique de la qualité globale que la mesure de la qualité ainsi que son évaluation doivent s'orienter non pas vers la recherche du meilleur et du pire, mais vers le plus probable. En tenant ce postulat pour acquis, un établissement (un programme, un service ou une intervention) constitue l'entité jugée la plus probable ou la plus porteuse pour atteindre les finalités qu'elle s'est elle-même données ou que d'autres lui ont attribuées. La mesure, tout comme l'évaluation, joue donc un rôle dont la visée est fondamentale à cet égard : diminuer le facteur de risque de commettre des erreurs dans le parcours vers l'accomplissement des fins poursuivies.

La mesure de la qualité, avons-nous signalé plus haut, devrait se faire selon trois points de vue : celui de la qualité voulue, celui de la qualité rendue et celui de la qualité perçue. Ces trois points de vue doivent être pris en considération et appliqués à chacun des liens de conformité compris dans le modèle de la qualité idéale. De même, lorsqu'il s'agira de produire des indicateurs à partir de ce modèle, il faudra s'assurer de couvrir ces trois points de vue.

\section{La pertinence}

La pertinence se définit comme le lien de conformité entre les objectifs visés par l'établissement et les besoins auxquels il doit répondre.

Le besoin quant à lui se définit toujours relativement à un manque. Quand un besoin est manifesté ou anticipé, c'est qu'il existe un écart entre la condition actuelle et la condition souhaitée ou idéale (Tyler, 1950; Kaufman, 1969). En d'autres termes, le besoin, c'est la différence entre la situation telle qu'elle se présente actuellement et ce qu'elle devrait être.

Dans le cas plus particulier de la mesure de la qualité, le besoin pourrait se définir comme la " différence entre un standard désiré ou normalement accepté, se rapportant à une action, une situation, une position ou à un objet et ce qu'est en réalité cette action ou cet objet " (Plante, 1982). La mesure de la pertinence n'est possible qu'au moment où les besoins et les objectifs sont clairement définis, du plus général au plus particulier. 
Sans liens avec les besoins de la société, du milieu ou de la collectivité, un établissement verrait sa pérennité grandement menacée. Aussi un établissement trouve-t-il sa principale raison d'être dans les liens qu'il entretient avec l'environnement humain dans lequel il baigne.

\section{La cohérence}

La cohérence se définit comme le lien de conformité ou de rapport logique et harmonieux entre les divers éléments qui composent un établissement. Les objectifs de l'établissement étant jugés en conformité avec les besoins auxquels il est censé répondre (la pertinence), les autres éléments constitutifs de l'établissement doivent s'unir et collaborer à l'atteinte de ceux-ci.

Mesurer cette qualité spécifique qu'est la cohérence, c'est établir la force des liens entre les divers moyens et les personnes qui composent un établissement et qui s'unissent les uns aux autres pour constituer un seul et même outil au service de l'atteinte des objectifs visés. Le but visé ici est de justifier la présence et la conformité de chacun de ces éléments afin d'apporter, le cas échéant, les corrections qui s'imposent auprès de certains d'entre eux pour constituer un tout cohérent.

\section{L'à-propos}

L'à-propos se définit comme le lien de conformité entre les diverses composantes de l'établissement et les contraintes auxquelles il est assujetti.

Des contraintes diverses et de toute nature influent sur un établissement. Ces contraintes sont extérieures à l'établissement ou intérieures, parce qu'elles sont fixées hiérarchiquement, sous formes de budgets ou autres, aux départements, services ou programmes qui le composent. Même si certaines contraintes ont un caractère intérieur à l'établissement, elles peuvent être considérées comme extérieures à ses départements, services et programmes. Les contraintes ont comme propriétés de limiter la marge de manœuvre d'un établissement et de devenir des conditions incontournables auxquelles doit se conformer un établissement ou l'une de ses parties constituantes.

Mesurer l'à-propos d'un établissement ou de l'une de ses parties, c'est donc établir les liens de conformité entre l'établissement et les 
contraintes qu'il a l'obligation de respecter. Ces conditions peuvent être d'ordre financier, physique, culturel, psychologique, intellectuel, politique, social ou autre.

À titre d'illustration de l'importance de se préoccuper de l'à-propos, pensons à l'âge ou à la culture des clients d'une fabrique de vêtements. Les vêtements peuvent répondre aux besoins des personnes visées, mais en même temps s'avérer non à propos par rapport à la culture de ces personnes ou à leur âge. De même, un service d'aide aux femmes monoparentales peut se révéler tout à fait pertinent par rapport aux besoins de ces femmes, tout en étant non à propos parce que ce service est offert à des heures qui ne leur conviennent pas. Un autre genre de service, bien qu'il soit pertinent, pourrait être gêné par des contraintes telles les distances, la disponibilité des moyens de transport, les communications. Bref, est considéré comme contrainte ce au regard de quoi l'établissement - ou l'une de ses parties - ne possède aucune marge de manœuvre pour changer la situation, mais à quoi il doit néanmoins se conformer au risque d'un mauvais fonctionnement. Toutes ces contraintes sont susceptibles d'avoir un effet sinon sur l'existence même de l'établissement, du moins sur son efficacité, son efficience et même son impact. La connaissance des contraintes auxquelles doit se soumettre un établissement ou l'une de ses parties (département, service, programme, etc.) s'avère donc des plus utiles au déroulement harmonieux de son fonctionnement.

\section{L'efficacité}

L'efficacité se définit comme le lien de conformité entre les objectifs visés par l'établissement et les résultats effectivement obtenus. En d'autres termes, elle peut être considérée comme l'expression du degré d'atteinte des objectifs réellement visés. Elle peut aussi être considérée comme le nombre d'objectifs effectivement atteints parmi les objectifs visés ou encore selon les deux points de vue à la fois.

Selon plusieurs auteurs, l'efficacité se révèle souvent une réalité difficile à définir. Elle apparaît comme une réalité insaisissable, qui résiste à la mesure. De façon générale, c'est aux résultats que l'on juge l'efficacité. Dans plusieurs circonstances, il y a lieu de s'interroger sur la nature des résultats à mesurer au sein d'un établissement. La plupart du temps, cela s'explique par l'absence d'objectifs explicites. Un objectif, rappelons-le, est un énoncé qui décrit le ou les buts que l'on se propose d'atteindre. 
La formulation d'objectifs très précis ou très opérationnels constitue une aide indéniable à la mesure sous l'angle de sa pertinence et de sa cohérence. Elle s'avère d'une importance tout aussi vitale lorsqu'il s'agit de mesurer l'efficacité d'un établissement, en soulevant la question de la conformité des résultats obtenus avec les objectifs visés. Elle se définit comme le degré d'atteinte des objectifs visés.

\section{La durabilité}

La durabilité se définit comme le lien de conformité entre les objectifs visés par un établissement et le maintien dans le temps des résultats obtenus. II s'agit ici, en quelque sorte, d'une sous-dimension de l'efficacité. Alors que cette dernière met en jeu les résultats immédiatement attribuables à l'établissement en lien avec les objectifs visés, la durabilité, parce qu'elle se situe dans le prolongement de l'efficacité, s'intéresse à la permanence de ces mêmes résultats.

Cette qualité s'avère particulièrement importante dans le cas des organismes voués aux services à la collectivité, notamment les établissements de santé et de services sociaux, les écoles, les cliniques ou tous les autres organismes visant l'acquisition et la maîtrise, à moyen et à long terme, de comportements, d'attitudes et de valeurs. Une baisse trop importante ou trop rapide des résultats devrait alors amener l'établissement à s'améliorer sous l'angle de cette qualité spécifique.

\section{L'efficience}

L'efficience se définit comme le lien de conformité entre l'économie des ressources réalisée et le degré d'atteinte des objectifs visés.

Un établissement dont l'efficacité a été démontrée est jugé efficient s'il lui est possible de satisfaire à l'une ou l'autre des conditions suivantes:

- Augmenter le degré d'atteinte des objectifs visés sans accroître les moyens alloués dans le fonctionnement pour y parvenir.

- Diminuer les moyens alloués dans le fonctionnement sans diminuer le degré d'atteinte des objectifs visés.

Ainsi définie, l'efficience est en relation de conformité avec un contexte d'économie. Cette économie peut s'exprimer par une diminution de 
temps, par une augmentation qualitative ou quantitative des résultats, par un nombre d'effets plus grands dans un temps donné ou par tous les autres changements qui, finalement, sont susceptibles de se traduire par une économie généralement financière.

Comme l'efficience ne doit aucunement altérer l'efficacité, cette qualité spécifique ne peut être considérée que dans le cas où un établissement a déjà fait la preuve, ou est en voie de la faire, de son efficacité.

\section{Le rendement}

Le rendement se définit comme le lien de conformité entre le potentiel humain que possède un établissement pour atteindre les objectifs visés et les résultats effectivement obtenus.

Le concept de rendement dont il est question ici doit être compris dans un sens particulier. II existe en effet une grande diversité de définitions de ce concept. Terme polysémique, l'expression qui s'y apparente le plus est celle de la performance. La performance ellemême s'entend souvent dans le sens de moteur, de mouvement, de ce qui bouge pour produire un résultat. C'est donc à la partie vivante d'un établissement que nous associons le rendement dans le modèle que nous présentons.

Dans un sens technique, pour confirmer le rôle de moteur que connote le rendement, celui-ci se définit ici comme la différence entre le potentiel qui caractérise un établissement et le frottement au sein de cet établissement. Moins il y aura de frottement entre les personnes qui travaillent dans un établissement, plus il y aura de synergie investie dans la production des résultats à meilleurs coûts.

Le rendement est une qualité spécifique qui, en lien de conformité avec un contexte d'économie, relève de préoccupations de l'ordre de l'efficience. À la gestion économique des choses le rendement ajoute la gestion des personnes comme condition nécessaire à la recherche de l'efficience.

Le potentiel de l'établissement nous est fourni principalement par des informations qui concernent, notamment, la compétence des personnes qui travaillent au sein de l'établissement, leur expérience, leurs réalisations, leurs succès sur les plans administratif et professionnel, leur capacité à accomplir une tâche donnée, etc. 
Le frottement, quant à lui, relève la plupart du temps de la personnalité des personnes en place, de l'ambiance de travail, du climat organisationnel, de la satisfaction à l'égard des tâches à réaliser, de la confiance, de la collaboration entre les personnes, de la transparence des communications, bref, de tout ce qui relève de la nature humaine et que l'on pourrait aussi qualifier d'hommerie.

La mesure du rendement constitue donc une occasion unique de se pencher de manière attentive sur l'un des éléments sans doute les plus importants d'un établissement : les personnes qui, à divers titres, font qu'un établissement est dynamique ou non, est prospère ou non.

\section{L'impact}

L'impact se définit comme le lien de conformité entre les résultats attribuables à l'établissement, mais non visés de façon explicite, et les exigences sociales, économiques, sociétales, physiques, psychologiques et autres de l'environnement dans lequel agit et interagit l'établissement.

Paul Valéry disait: "Essayez de penser à un arbre sans le situer dans un environnement. "C'est là un exercice qui se révèle pratiquement impossible. Il en est de même pour un établissement qui existe en raison de la qualité du rapport qu'il entretient avec les besoins de la société, du milieu et de la collectivité. Parce qu'il occupe une place physique dans un milieu, engendre des activités, l'établissement est source d'une multiplicité d'échanges plus ou moins harmonieux avec l'environnement physique et humain dans lequel il s'insère. Ces échanges favorisent l'émergence d'effets qui, tout en étant tout à fait étrangers à ceux qui sont attendus à travers les objectifs, sont parfois très favorables à l'établissement, mais aussi, parfois, entièrement défavorables.

L'impact englobe l'ensemble des effets attribuables à l'établissement, mais non visés par celui-ci. Cette qualité spécifique qu'est l'impact prend en considération la nature des effets non prévus de l'établissement en lien de conformité avec les divers environnements avec lesquels il est en contact. II peut s'agir ici d'un environnement physique, social, familial, organisationnel, économique, politique, religieux, psychologique, etc. 


\section{La flexibilité}

La flexibilité se définit comme le lien de conformité entre les capacités d'amélioration que possède un établissement et les lieux où des améliorations s'imposent au sein de cet établissement.

La possession de cette qualité est garante de l'utilité de la mesure des autres qualités à la prise de décision. À ce titre, il vaut la peine de s'y attarder plus longuement. Selon Gerwin (1987), il est fréquent dans les organisations de constater que " part of the problem arises from the lack of operational measures of flexibility ». Pour Cox (1989), la flexibilité doit être considérée " as a measure of the efficiency with which the manufacturing process can be changed ". La flexibilité est la qualité spécifique que présente un établissement qui possède les moyens nécessaires et appropriés pour apporter des changements là où ils s'imposent. La flexibilité étant ainsi définie, deux conditions doivent être respectées pour la mesurer :

- Avoir la capacité de s'améliorer, ce qui ne peut se déterminer qu'à la lumière de l'importance des moyens que possède ou que peut investir un établissement ou un système pour apporter des changements.

- Avoir la connaissance des points à améliorer, ce qui ne peut être connu qu'à la lumière du degré d'amélioration qui doit être apporté aux constituants importants de l'établissement.

Ces deux conditions varient en intensité. Tant la capacité d'amélioration que le degré d'amélioration peuvent être grands, moyens, petits ou nuls. Comme l'illustre le tableau ci-dessous, cinq types de flexibilité sont possibles au sein d'un établissement.

Il y a d'abord une flexibilité en équilibre lorsque le degré d'amélioration à apporter à un élément important varie selon la même intensité que la capacité d'amélioration que possède un établissement au regard de cet élément. L'établissement dispose alors des moyens de s'ajuster aussitôt que le besoin de changement se fait sentir.

Dans le cas où des améliorations doivent être apportées et où l'établissement possède une capacité d'amélioration égale ou supérieure à la situation d'équilibre, il y a présence d'une flexibilité efficiente. 


\section{Type de flexibilité}

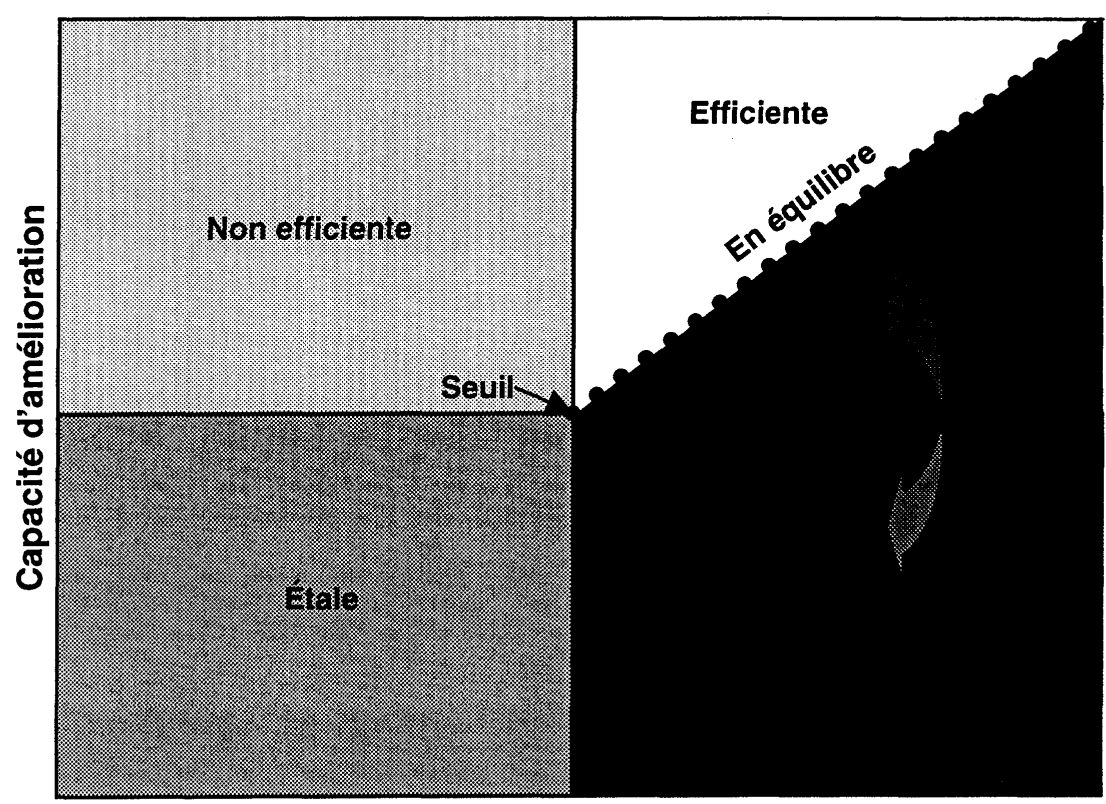

Degré d'amélioration

(C) Jacques Plante et Chantal Bouchard, 2000

Si un établissement est dans une situation inverse, c'est-à-dire que sa capacité d'amélioration au regard d'un élément particulier est sous le seuil d'équilibre, il y a alors flexibilité en rupture, ce qui est le signe d'un problème qui peut être sérieux.

Dans le cas où un établissement fait la preuve d'une capacité d'amélioration notable au regard d'éléments qu'il n'est pas important d'améliorer ou qui ont peu besoin d'amélioration, il y a alors flexibilité non efficiente. Dans cette dernière situation, il faudrait vérifier s'il est possible que ce type de surcapacité puisse être transformé en flexibilité efficiente.

Enfin, il y a flexibilité étale lorsqu'il y a à la fois absence ou peu de capacité d'amélioration de certains éléments importants au sein d'un établissement et peu ou pas d'amélioration à apporter à ces mêmes éléments. 
La flexibilité est donc cette qualité spécifique nécessaire que possède un établissement de pouvoir s'améliorer là où il doit s'améliorer. Sans la possession d'un certain degré de flexibilité, il peut être peu rentable de procéder à la mesure des autres qualités de l'établissement. À quoi servira-t-il en effet de connaître le degré de possession des autres qualités si l'établissement n'a pas en lui la flexibilité suffisante pour s'améliorer?

Deux types d'information nous semblent nécessaires pour mesurer la flexibilité. II faut d'abord savoir quel est le degré d'amélioration à apporter à l'un ou l'autre ou à l'ensemble des éléments qui composent un établissement. II faut ensuite connaître la capacité d'amélioration que possède cet établissement pour s'améliorer, c'est-à-dire la possession plus ou moins grande, par l'établissement, des moyens spécifiques qui peuvent être mis en œuvre ou investis pour élever la qualité de ces éléments. Dans un cas comme dans l'autre, des échelles de mesure relativement simples peuvent être utilisées avec profit pour déterminer les valeurs propres à ces deux composantes de la flexibilité d'un établissement.

\section{VERS L'ÉTABLISSEMENT DES INDICATEURS DE LA QUALITÉ}

Certains phénomènes ou objets sont plus difficiles que d'autres à définir, donc à mesurer. II faut dès lors rechercher des signes de ces phénomènes ou de ces objets. En d'autres termes, cela revient à établir un continuum signifiant-signifié où la somme des signes observés permet de témoigner de l'objet ou du phénomène signifié. Certes, l'utilisation d'indicateurs en lieu et place de la mesure directe d'un objet comporte le danger d'une perception réductionniste de l'objet ou du phénomène observé. Les propos fort connus du physicien Kelvin (1824-1907), créateur de l'échelle pour mesurer le degré de chaleur d'un corps, sont toujours valides aujourd'hui. En substance, disait-il, " when you can measure what you are speaking about, and express it in numbers, you know something about it... otherwise your knowledge is of a meagre and unsatisfactory kind; it may be the beginning of knowledge, but you have scarcely in thought advanced to the stage of science ".

La qualité est certes une réalité que l'on peut classer facilement parmi les objets ou les phénomènes qui échappent à une mesure directe. II 
n'est alors pas étonnant de constater que, chez la majorité des auteurs consultés, on fait appel à des indicateurs de la qualité. C'est actuellement le meilleur moyen dont on dispose pour tenter d'en circonscrire la valeur.

L'utilité première des indicateurs est de servir de base à l'analyse de l'état de la qualité d'un établissement dans son entier ou de l'une ou l'autre de ses parties. De la même manière que la qualité fait maintenant partie intégrante du vocabulaire commun des décideurs, l'indicateur occupe aujourd'hui une place prépondérante dans le répertoire terminologique des décideurs.

C'est dans le domaine économique que l'on a observé l'utilisation première et à grande échelle du terme indicateur. L'utilisation des indicateurs pris individuellement ou regroupés (indices) s'est répandue rapidement, puisque ces indicateurs ont permis de suivre l'évolution de l'économie d'une manière quantitative. Les buts visés étaient de trois ordres : mesurer l'état et le changement d'une réalité, fournir aux gestionnaires et décideurs une base pour juger l'état de santé d'un système ou d'une organisation et servir de source de réflexions afin de réorienter le système ou l'organisation, le cas échéant.

Des spécialistes d'autres disciplines, telles les sciences sociales, les sciences de l'éducation, les sciences de la santé et les sciences de l'administration, se sont joints par la suite aux économistes en empruntant cette notion d'indicateur. Actuellement, les indicateurs sont utilisés dans tous les secteurs d'activités industrielles et de services.

Dans l'élaboration de nouvelles conceptions de la gestion comme la gestion par objectif et la gestion axée sur les résultats, nous dit McDaniel (1996), les indicateurs " constituent une source essentielle d'information et d'inspiration ».

L'utilisation à grande échelle des indicateurs est souvent critiquée. Déjà, en 1978, Richard M. Jaeger invitait à la prudence. Des centaines d'articles et de rapports ainsi que des douzaines de livres sur les indicateurs dans tous les domaines ont amené, selon Jeager, à associer abusivement le concept d'indicateur à celui de résultats statistiques, dont les applications possibles, notamment en éducation et en santé, font l'objet de vives discussions et alimentent des controverses. 
Aujourd'hui encore, à la lecture des nombreuses publications sur le sujet, il n'est pas évident que ce concept ait acquis un caractère univoque tant dans sa définition que dans son utilisation et dans son interprétation. Même si l'ensemble des utilisateurs semblent se rallier autour de certaines expressions telles « indicateurs de performance ", " indicateurs de l'efficacité ", « indicateurs de résultats » " indicateurs de l'impact » et « indicateurs de processus ", l'interprétation de chacune de ces expressions varie d'un établissement à l'autre, d'un système à l'autre et même d'un pays à l'autre (McDaniel, 1996).

\section{La définition d'un indicateur}

Un indicateur se définit comme tout événement quantitatif ou qualitatif qui témoigne de l'état d'un autre événement qui ne peut être mesuré directement ou objectivement. C'est donc en sa qualité de témoin qu'un indicateur doit être interrogé en premier lieu. II ne suffit pas de croire à sa valeur, il faut la démontrer.

Les ouvrages traitant des indicateurs fournissent un éventail assez large des définitions possibles. Plusieurs prennent pour référence les caractéristiques que doit posséder un indicateur, alors que d'autres s'appuient sur certaines de leurs particularités. Quelques définitions encore tiennent compte de typologies ou même de certaines modalités qu'on croit nécessaires à la description exacte de ce concept. II est difficile ici de donner un véritable aperçu de la diversité des définitions données au concept d'indicateur. Les quelques définitions que nous retiendrons émanent de divers secteurs d'activité; elles présentent la notion d'indicateur sans référence à un champ d'application en particulier. Bien que peu nombreuses, elles rendent compte, selon nous, des définitions que nous avons trouvées dans les nombreux ouvrages spécialisés que nous avons consultés et qui traitent de ce sujet. À la lumière de ces quelques définitions, il nous sera possible de dégager des caractéristiques qui semblent communes à lindicateur et sur lesquelles nous pourrons nous appuyer pour clarifier ce concept.

Le Mouvement français pour la qualité (1992) associe le concept d'indicateur à celui de la qualité. Rattaché à un phénomène, un indicateur est " une information choisie destinée à en observer périodiquement les évolutions par rapport à des objectifs fixés ". Dans le document que ce même groupe a publié deux ans plus tard sous le titre de 
Synthèse du groupe de travail - indicateur qualité et tableau de bord, on le définit cette fois-ci non plus comme une information, mais plutôt comme un outil associé à un phénomène qui permet de l'observer et de le contrôler (1994, p. 33). Il exprime la qualité perçue par le client à travers des services effectivement offerts. II décrit également la qualité interne des services par l'entremise des processus suivis pour les obtenir. Il exprime enfin l'amélioration de la qualité vue au moyen des efforts déployés.

Saucier et Brunelle (1995) distinguent le concept d'indicateur de celui de descripteur. Un descripteur constitue une mesure directe d'un phénomène. À titre d'exemple, pensons aux taux de naissance dans une population pour établir l'accroissement d'une population ou d'une souspopulation. L'indicateur, quant à lui, a valeur de référent lorsqu'il est utilisé comme point de repère dans l'évaluation de l'état ou de l'évolution d'un phénomène donné, non quantifiable directement. C'est le cas par exemple de l'intelligence ou des attitudes pour lesquelles on doit faire appel à des indicateurs pour mieux les estimer. Un indicateur ne devient tel qu'à partir du moment où il devient une information significative par rapport au phénomène observé pour la personne qui le consulte. Ainsi, une statistique, un chiffre, une donnée ne constituent pas automatiquement des indicateurs. II doit d'abord exister une association claire entre le phénomène observé et l'élément mesuré.

Dans son Dictionnaire de l'évaluation et de la recherche en éducation Gilbert De Landsheere (1979) accorde à l'indicateur un rôle de témoin. Est indicateur « tout phénomène témoignant de l'existence d'un autre ". Selon lui, on recherche généralement des indicateurs spécifiques et aisément mesurables. Ces indicateurs peuvent être soit des échantillons des phénomènes ou événements mesurés, soit des symptômes. En reprenant son exemple pour illustrer ce qu'il entend par symptôme, la longueur moyenne des mots, exprimée en lettres, constitue un indicateur d'abstraction. II ajoute de plus que les nombreuses entités hypothétiques (constructs) utilisées par la psychologie sont construites à partir de l'observation des indicateurs. Et il n'est pas exceptionnel que la validité des indicateurs retenus pour mesurer un trait ou une situation soit insuffisamment établie.

Brunelle (1993) voit dans l'indicateur une obligation de lien implicite et même d'évidence entre la situation observée et l'élément utilisé pour le juger. Un indicateur est « un signal permettant de postuler que des 
résultats effectifs observés sont supérieurs, conformes ou inquiétants par rapport à ce qui était attendu " (Brunelle, 1993, p. 14). Sous plusieurs aspects, cette définition rejoint celle de Madeleine Grawitz (1993), auteure réputée dans le domaine des sciences sociales, pour qui un indicateur est une donnée observable permettant d'appréhender les dimensions, la présence ou l'absence de phénomènes que l'on ne peut saisir directement ni objectivement. Elle ajoute cependant dans son ouvrage que "le plus intéressant dans les sciences sociales relève de la qualité, c'est-à-dire d'éléments difficiles à mesurer " (Grawitz, 1993, p. 335). Mais il ne s'agit pas ici d'opposer le qualitatif au quantitatif, puisque ce dernier, pour se prêter à l'acte de mesurer, dépend souvent plus ou moins directement de la qualité. Par ces propos, Grawitz (1993) souligne que l'on ne peut mettre en opposition l'aspect qualitatif et quantitatif des indicateurs. Guthrie (1987) est du même avis au moment où il signale cette dualité. Ce ne sont pas les indicateurs en eux-mêmes qui sont qualitatifs, mais « the dual traits of instructional effectiveness... its quantity and quality... both should be contained in a full model of $y$ ". En d'autres termes, indicateur qualitatif et indicateur quantitatif ne sont pas des réalités différentes, mais plutôt les deux côtés d'une même médaille.

Ces quelques définitions, croyons-nous, constituent un échantillon représentatif du type de définition habituellement prêté à un indicateur. Elles rendent compte d'une pluralité de points de vue, certes, mais font néanmoins montre d'un large consensus quant à la nature d'un indicateur. Tous les auteurs consultés s'entendent pour reconnaître à l'indicateur une valeur de témoignage. Vu sous cet angle, l'indicateur devient un repère utile et tangible à partir duquel, additionné à d'autres indicateurs, il est possible de surveiller le fonctionnement global ou détaillé d'un système, de même que son cheminement vers l'atteinte des objectifs fixés. En bref, les indicateurs permettent d'inférer la qualité du système tout entier. Les éléments significatifs que l'on peut extraire des diverses définitions attribuées ci-dessus au concept d'indicateur semblent se résumer aux faits qu'un indicateur

- n'est pas un descripteur de la réalité, il en témoigne (De Landsheere, 1979);

- est utile surtout lorsqu'on veut témoigner de l'état d'un phénomène difficile à saisir directement et objectivement (Grawitz, 1993); 
- a comme raison d'être d'informé sur l'état de santé d'un système, qu'il s'agisse d'une organisation, d'un programme ou d'un service (Saucier et Brunelle, 1995);

- doit correspondre à un certain nombre de caractéristiques pour être de qualité (McDaniel, 1996);

- doit être associé fortement au phénomène dont il témoigne.

En investissant ces cinq éléments au profit de la définition d'un indicateur, on peut conclure que celui-ci peut se définir comme tout événement qualitatif ou quantitatif qui témoigne d'un autre événement impossible à mesurer directement et objectivement. Appliqué au contexte de la mesure de la qualité, un indicateur doit être clairement associé à une ou des qualités préalablement déterminées pour assumer de façon valide sa fonction de témoin.

Par événement qualitatif ou quantitatif, entendons ici une donnée statistique, un rapport, un taux, le résultat d'un comptage, un ratio, un indice (addition de plusieurs indicateurs), une notation, un facteur de risque, une valeur, une attitude, etc.

La formulation d'un indicateur peut donc prendre la forme d'un simple nombre, d'un rapport entre deux quantités de même unité mais de nature différente ou encore d'un rapport entre deux ou plusieurs quantités d'unités différentes, d'indice global, de coûts, de profits, de pertes, de composition arithmétique de plusieurs indicateurs, de degré de satisfaction ou de contentement sur une échelle d'appréciation prédéterminée, de probabilité, de degré d'attitude, de comportement, de type de valeur, de préférence ou autres.

Pour ce qui est d'événements impossibles à mesurer directement et objectivement, pensons à l'ambiance de travail, à un processus, à la gestion, à un service ou encore à l'une ou l'autre des neuf qualités spécifiques comprises dans le modèle schématique de la qualité globale présenté plus haut.

\section{LA DÉFINITION DES QUALITÉS D'UN INDICATEUR DE LA QUALITÉ}

Nous venons de le voir, il existe plusieurs définitions du concept d'indicateur. Les acceptions du terme varient d'un auteur à l'autre, mais elles véhiculent sensiblement le même sens : un indicateur a pour 
fonction première de servir de témoin. Ainsi, à l'instar de tout témoin, il se doit de posséder un certain nombre de qualités pour être jugé crédible et ainsi devenir utile.

Nous devons être particulièrement attentifs lorsqu'il s'agit de faire état des qualités et du nombre de qualités que doit posséder un indicateur. II est facile en effet de confondre l'expression indicateur de qualité avec la fonction d'un indicateur de la qualité dont le rôle est de témoigner de la qualité voulue, rendue ou perçue.

Ainsi, par exemple, il peut exister une nette différence entre des indicateurs de gestion et des indicateurs de la qualité. Force nous est de constater que ce point de vue n'est pas partagé par l'ensemble des gestionnaires. Une partie importante des gestionnaires est encore encline à penser que la quantité est plus facile à vendre que la qualité.

Aussi, bien souvent, les indicateurs retenus se ramènent à des expressions de rapports ou de taux de flux de clientèle, de nombre d'usagers d'un service, de nombre d'usagers par unité de temps, de nombre de services offerts, etc. Pour une bonne part, ces indicateurs se limitent à témoigner de la gestion des organismes, c'est-à-dire de la présence d'un objet particulier et non de la qualité de cet objet.

Même si l'on estime que témoigner de la présence d'un objet n'est pas un phénomène à négliger au sein d'un organisation, deux questions demeurent. Est-ce réellement de la présence de cet objet et uniquement de cette présence qu'on veut témoigner ou est-ce de la qualité ou des qualités que possède cet objet? Dans un contexte de mesure de la qualité et de son amélioration, où impérativement nous devons définir les qualités attendues, quel est ou quels sont les indicateurs qui peuvent le mieux témoigner de la conformité des objets examinés?

Olaf C. McDaniel fait rapidement le tour des qualités que doivent posséder des indicateurs lorsqu'il affirme qu'ils ne sont utiles que dans le cas où ils "sont suffisamment pertinents, vérifiables, quantifiables, acceptés par les établissements et peu onéreux à exploiter 》 (1996, p. 137). Saucier et Brunelle précisent qu'un indicateur, pour être de qualité, doit présenter une validité démontrée et une robustesse, c'està-dire une « capacité à résister à l'utilisation que l'on en fait » (1995, p. 25). Parmi les autres attributs que doit posséder un indicateur, il identifie la disponibilité des sources, la qualité des sources, l'utilité, 
l'intelligibilité, sa capacité d'être ventilé selon divers niveaux, sa précision et son coût raisonnable d'utilisation.

Deux aspects doivent être considérés ici pour juger de la crédibilité d'un indicateur : l'aspect technique de l'indicateur et son aspect qualitatif. Sous ces deux aspects, on retrouve sensiblement les mêmes caractéristiques qui font qu'un instrument de mesure, à quelque domaine qu'il appartienne, devient un instrument de qualité.

Sous l'angle technique, un indicateur doit :

- permettre de mesurer ou d'estimer les caractéristiques quantitatives et qualitatives d'un objet, d'une situation, d'une action ou d'un événement significatif au sein d'un organisation, d'un programme ou d'un service;

- mesurer des traits ou des réalités durables et persistantes du système de manière que des tendances et des changements puissent être mesurés dans le temps;

- être comparables dans le temps avec des standards ou avec des normes;

- fournir un point de repère permettant de mesurer dans le temps une stabilité, une progression ou une régression intra-organisation, intra-programme ou intra-service, de même que, le cas échéant, une stabilité, une progression ou une régression interorganisations, inter-programmes et inter-services.

Sous son aspect qualitatif, un indicateur doit être :

- valide, c'est-à-dire témoigner réellement de l'état de l'événement dont il est censé témoigner. Trois qualités supplémentaires se conjuguent à la validité :

- la fiabilité, c'est-à-dire le pouvoir de fournir une image exacte de l'événement observé,

- la fidélité (stability), c'est-à-dire le fait de fournir la même information lorsque l'indicateur est utilisé de manière répétée dans les mêmes conditions pour un événement qui ne change pas,

- la sensibilité, c'est-à-dire la variation de la valeur indiquée par l'indicateur parallèlement aux changements de l'état de l'événement observé; 
- opérationnel, c'est-à-dire facile à établir et à formuler :

- facile à utiliser,

- crédible aux yeux des utilisateurs et du public concerné,

- acceptable par les utilisateurs et le public concerné,

- compréhensible par un vaste auditoire;

- agrégeable, c'est-à-dire facile à quantifier :

- peut être regroupé sous forme d'indices le cas échéant,

- est facile à regrouper et à cumuler en vue d'une synthèse,

- est facile à regrouper et à cumuler en vue d'une analyse;

- économique, c'est-à-dire chiffrable pécuniairement :

- utile à la prévention,

- utile à la prise de décision.

Ces caractéristiques peuvent être utilisées avec profit à titre de guide ou de grille permettant de vérifier la qualité des indicateurs choisis au sein d'un organisation. Mais, est-il utile de le rappeler, dans le contexte précis de la mesure de la qualité, le fait de disposer d'indicateurs de qualité ne signifie pas que l'on dispose automatiquement d'indicateurs de la qualité.

\section{CONCLUSION}

Être de qualité, nous l'avons vu, c'est la caractéristique que possède un établissement lorsqu'il est conforme à un standard ou à une norme préalablement définis. La mesure de la qualité comporte donc plusieurs obstacles à franchir. Le premier est, nous l'avons vu, celui de définir clairement et précisément ce qu'on a l'intention de mesurer. Sans cette condition sine qua non, il n'est pas possible d'assurer la validité de la mesure.

Dans cet article relativement théorique, nous avons présenté un modèle schématique de la qualité globale qui répond aux canons de la spécialité en mesure et évaluation et qui peut être utilisé comme référent pour déterminer des indicateurs de la qualité. La mesure des neuf qualités spécifiques comprises dans ce modèle permet de prendre en 
compte les changements qui s'opèrent ou qui doivent s'opérer au sein d'un établissement. Ces qualités spécifiques, mesurées individuellement, constituent autant de détecteurs de fumée susceptibles de nous informer du bon ou du mauvais fonctionnement de certaines parties de l'établissement. C'est de cette manière, selon nous, que nous pouvons le mieux établir la différence entre un simple changement et une amélioration de la qualité qui, elle, suppose un changement dans une direction voulue, rendue et perçue. II reste maintenant à appliquer ce modèle à l'évaluation sur le terrain de la qualité d'un établissement de santé et de services sociaux².

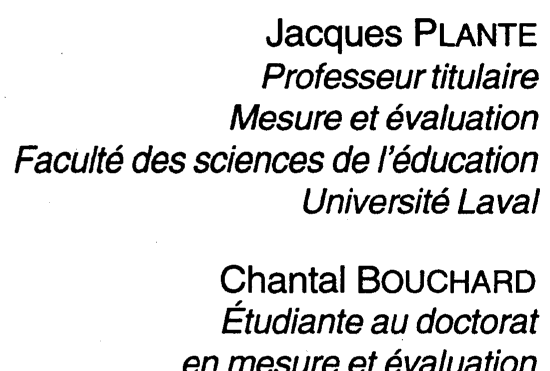

Certificat en gestion et assurance de la qualité

Conseillère cadre au développement Conseil québécois d'agrément d'établissements de santé et de services sociaux

\section{Références bibliographiques}

ALKIN, M.C. (1969). " Evaluation Theory Development ", Evaluation Comment, vol. 2, no 1, p. 2-7.

BEMOWSKI, K. (1992). “ The quality glossary », Quality Progress, vol. 25, n², p. 18-29.

BRUNELLE, Y. (1993). La qualité des soins et services : un cadre conceptuel. Études et analyses, Ministère de la Santé et des Services sociaux, Direction générale de la planification et de l'évaluation, Québec.

2. Ce sera là l'objet principal d'un article à paraître ultérieurement. 
COMMISSARIAT GÉNÉRAL DU PLAN ET ÉCOLE NATIONALE D'ADMINISTRATION (ENA) (1995). Principes d'une évaluation socialement utile, Paris, La Documentation française, vol. Outils, Pratiques, Institutions pour évaluer les politiques publiques, Paris.

CONSEIL SCIENTIFIQUE DE L'ÉVALUATION (1993). L'évaluation en développement, Rapport annuel sur l'évolution des pratiques d'évaluation des politiques publiques, Paris.

Cox, T. (1989). " Towards the measurement of manufacturing flexibility ", Productivity and Inventory Management, vol. 30, no 1, p. 68-72.

CRAWFORD, K.M. et J.F. Cox (1990). « Designing performance measurement systems for just-in-time operations ", International Journal of Production Research, vol. 28, no 11, p. 25-36.

Crosby, P.B. (1992). Completeness. Quality for the 21st Century, Dutton, U.S.A. CrosBY, P.B. (1987). Quality without Tears, Singapour, McGraw-Hill.

Crosby, P.B. (1979). Quality Is Free, New York, NY, McGraw-Hill.

CusINS, P. (1994). “ Understanding Quality through Systems Thinking ", The TQM Magazine, vol. 06, $\mathrm{n}^{\circ}$ 5, p. 19-27.

DALE, B.G. (dir.) (1994). Managing Quality, Englewood Cliffs, NJ, Prentice-Hall. DALE, B.G., R.J. BOADEN et D.M. LASCELLES (1994). « Total quality management : an overview ", dans B.G. Dale (dir.), Managing Quality, Englewood Cliffs, NJ, Prentice-Hall, p. 3-40.

DE LANDSHEERE, G. (1979). Dictionnaire de l'évaluation et de la recherche en éducation, Paris, Presses universitaires de France, $338 \mathrm{p}$.

DEMING, W.E. (1986). Out of the Crisis, Boston, MA, MIT.

Deming, W.E. (1982). Quality, Productivity and Competitive Position, Cambridge, Massachusetts Institute of Technology.

Dictionnaire actuel de L'ÉdUCATION (1993). (2éd.), Montréal, Guérin et Paris, Eska.

Dixon, J.R., A.J. NANNI et T.E. Vollmann (1990). The New Performance Challenge - Measuring Operations for World-Class Competition, Homewood, III., Dow Jones-Irwin.

EFQM (1997). Self-assessment. Guidelines for Companies, Pays-Bas, Pabo Print BV.

Feigenbaum, A.V. (1991). Total Quality Control ( $3^{e}$ éd. rév.), New York, McGraw-Hill.

FeIgenbaum, A.V. (1961). Total Quality Control, New York, McGraw-Hill.

FeIGENBAUM, A.V. (1956). « Total quality control », Harvard Business Review, vol. 34, n 6, p. 93-101. 
FORTUIN, L. (1988). "Performance indicators - why, where and how? ", European Journal of Operational Research, vol. 34, no 1, p. 1-9.

GERWIN, D. (1987). " An agenda of research on the flexibility of manufacturing processes ", International Journal of Operations \& Production Management, vol. 7, no 1, p. 38-49.

GILMORE, H.L. (1974). “ Product conformance cost », Quality Progress, vol. 7, n० 5, p. 16-19.

GLOBERSON, S. (1985). " Issues in developing a performance criteria system for an organization », International Journal of Production Research, vol. 23, no 4, p. 639-646.

GraWITZ, M. (1993). Méthodes des sciences sociales (9e éd.), Paris, Édition Dalloz.

GUBA, E.G. et Y.S. LINCOLN (1981). Effective Evaluation, San Francisco, Jossey-Bass.

GUTHRIE, John T. (1987). Indicators of Reading Education, document non publié.

HALL, R.W. (1983). Zero Inventories, Homewood, III., Dow-Jones Irwin.

HAMOND, R.L. (1973). Evaluation at the local level, dans B.R Worthern et J.R. Sanders (dir.), Educational evaluation : theory and practices, Worthington, Ohio, C.A. Jone Publishing Compagny.

HARTE, H.G. et B.G. DALE (1995). « Improving quality in professional service organizations : a review of the key issues ", Managing Service Quality, vol. $05, n^{\circ} 3$.

HAUSER, J.R. et D. ClaUsing (1988). «The house of quality ", Harvard Business Review, vol. 66, n³, p. 63-73.

HousE, C.H. et R.L. PRICE (1991). « The return map : tracking product teams », Harvard Business Review, janvier-février, p. 92-100.

ISHIKAWA, K. (1990). Introduction to Quality Control, Tokyo, 3A Corporation.

ISHIKAWA, K. (1989). « How to apply companywide quality control in foreign countries ", Quality Progress, vol. 22, n 9, p. 70-74.

ISHIKAWA, K. (1985). What Is Total Quality Control? The Japanese Way, New York, Prentice-Hall.

JAEGER, R.M. (1978). « About education indicator; Statistics on the condition and trends in education ", Review of Research Education, p. 276-315.

JURAN, J.M. (dir.) (1995). A History of Managing for Quality, Milwaukee, Wisconsin, ASQC Quality Press.

JURAN, J.M. (1988). Juran on Planning for Quality, New York, Free Press. 
JuRAN, J.M., F.M. Gryna et R.S. BInGHam (dir.) (1974). Quality Control Handbook ( $3^{\mathrm{e}}$ éd. rév.), New York, McGraw-Hill.

JURAN, J.M. et F.M. GRYNA (dir.) (1988). Quality Control Handbook (4e éd. rév.), New York, McGraw-Hill.

KAPLAN, R.S. et D.P. NORTON (1992). " The balanced scorecard - measures that drive performance ", Harvard Business Review, janvier-février, p. 71-90.

KAUFMAN, R. (1969). Educational system planing, dans R. Kaufman (1972), Englewood Cliffs, NJ, Prentice-Hall Educational Administration Series.

LEA, R. et B. PARKER (1989). " The JIT spiral of continuous improvement », IMDS, vol. 4, p. 10-13.

LINCOLN, Y.S (1987). " L'évaluation de programmes en l'an 2000 : Problèmes et solutions ", dans M.A. Nadeau et M. Hurteau (dir.), L'évaluation : défis des années 80 , Monographie en mesure et évaluation, Département de mesure et évaluation, Université Laval, Québec.

MARTÍNEZ-LORENTE, ANGEL R. et collab. (1998). « Total quality management : origins and evolution of the term ", The TQM Magazine, vol. 10, $\mathrm{n}^{\circ} 5$.

MCDANIEL, O.C. (1996). Utilisation théorique et pratique des indicateurs de performance, Gestion de l'enseignement supérieur, vol. 8, n॰ 3 .

MORRISON, S.J. (1994). " Managing quality : an historical review », dans B.G. Dale (dir.), Managing Quality, Englewood Cliffs, NJ, Prentice-Hall.

MOUVEMENT FRANÇAIS POUR LA QUALITÉ (1994). Synthèse du groupe de travailindicateur qualité et tableau de bord, Paris.

MOUVEMENT FRANCAIS POUR LA QUALITÉ (1992). Indicateurs de qualité et tableau de bord, Paris

NEELY, A. (1999). " The performance measurement revolution : why now and what next? ", International Journal of Operations \& Production Management, vol. 19, $\mathrm{n}^{\circ} 2$.

NeEly, A.D., M. GREgory et K. PlatTs (1995). « Performance measurement system design : a literature review and research agenda ", International Journal of Operations \& Production Management, vol. 15, nº 4, p. 80-116.

NeERGAARD, P. (1999). " Quality management : a survey on accomplished results ", International Journal of Quality \& Reliability Management, vol. 16, n० 3, p. 227-289.

OAKLAND, J.S. (1993). Total Quality Management ( $2^{\mathrm{e}}$ éd.), ButterworthHeinemann, Oxford.

Parasuraman, A., V.A. Zeithaml et L.L. Berry (1985). « A conceptual model of service quality and its implications for further research ", Journal of Marketing, vol. 49, n 4, p. 41-50.

PATTON, M.Q. (1980). Qualitative Evaluation Methods, Beverly Hills, CA, Sage. 
PETERS, T.J. et R. WATERMAN (1982). In Search of Excellence : Lessons From America's Best-run Companies, New York, NY, Harper and Row.

PLANTE, J. (1994). Évaluation de programme (français, anglais, espagnol), Québec, Presses de l'Université Laval.

PLANTE, J. (1982). Étude de certaines variations des besoins exprimés en partie sur l'auto-évaluation (Thèse de doctorat, Université Laval).

POWELL, T.C. (1995). « Total quality management as competitive advantage : a review and empirical study ", Strategic Management Journal, vol. 16, $\mathrm{n}^{0}$ 1, p. 15-37.

Provus, M.D. (1971). Discrepancy Evaluation, Berkeley, CA, MacCutchan Publishing Corporation.

REEVES, C.A. et D. BEDNAR (1994). Defining quality : alternatives and implications, Academy of Management Review.

SAUCIER, A. et Y. BRUNELLE (1995). Les indicateurs et la gestion par résultats, Méthodologie et instrumentation, Québec, Ministère de la Santé et des Services sociaux, Direction générale de la planification et de l'évaluation.

SHERGOLD, K. et D.M. REED (1996). "Striving for excellence: how selfassessment using the Business Excellence Model can result in improvements in all areas of business activities ", The TQM Magazine, vol. 8, $n^{\circ} 6$, p. 48-52.

STAKE, R.E. (1967). « The Countenance of Educational Evaluation », Teachers College Record, vol. 68, p. 523-540.

StARCH, D. et E.C. ElLIOT (1912). "Rehability of Grading Work in Mathematics ", School Review.

STUELPNAGEL, T.R. (1993). « Déjà vu : TQM returns to Detroit and elsewhere », Quality Progress, vol. 26, n 9, p. 91-95.

StUfFLeBEAM, D.L., W.J. FoleY, W.J. GePHART et collab. (1971). Educational Evaluation and Decision Making, Istaca, III., Peacock.

TuiJnman, A.C. et T. Postlewaite NeVILle (1994). Monitoring the Standards of Education, Oxford, Angleterre, Pergamon Press.

TYLER, R.W. (1950). Basic Principles of Curriculum and Instruction, Chicago, III., University of Chicago Press.

UNESCO (1995). Guide d'évaluation d'un programme, Paris, UNESCO.

WILLIAMS, R.H. et R.M. ZIGLI (1987). "Ambiguity impedes quality in service industries », Quality Progress, vol. 20, n 7, p. 14-17. 